\title{
Filters for diagnostic of Doppler reflectometry on the L-2M stellarator for operation under conditions of high ECR heating power
}

\author{
D.V. Malakhov ${ }^{1,2,3}$, A.A. Kharchevsky ${ }^{1,3}$, N.N. Bogachev ${ }^{1,2,3}$, N.N. Skvortsova ${ }^{1,4}$ \\ ${ }^{1}$ Prokhorov General Physics Institute, Moscow, Russia, 89199945791@mail.ru \\ ${ }^{2}$ Pirogov Russian National Research Medical University, Moscow, Russia \\ ${ }^{3}$ Moscow Technological University (MIREA), Moscow, Russia \\ ${ }^{4}$ National Research Nuclear University MEPhI, Moscow, Russia
}

The study of the properties of edge plasma, in particular, the plasma rotation speed, in toroidal traps is associated with the search for ways to increase the efficiency and reliability of the thermonuclear reactor. One of the methods for measuring the rate of poloidal plasma rotation in tokamaks and stellarators is Doppler reflectometry (DR). The Doppler reflectometer is designed to work as part of the experimental L-2M device to measure the rates of its poloidal rotation, as well as to measure the spatialfrequency spectra of plasma density fluctuations [1].

Diagnostics of high-temperature plasma in magnetic confinement systems is associated with a variety of problems and requires atypical engineering solutions. The powerful sources of microwave radiation (gyrotrons) used to heat the plasma [2] interfere with the detection of low-power diagnostic signals. To isolate such signals, it is necessary to provide high suppression at the ECR frequency of heating $75 \mathrm{GHz}$ and to provide low attenuation at the diagnosis frequency of $30-40 \mathrm{GHz}$. The created system for filtering electromagnetic radiation consists of a band-stop filter based on a Fabry-Perot resonator and three, pin-shaped waveguide filters [3]. The CAD EMPro Keysight Technologies in a three-dimensional geometry of band-stop filter was represented as a few pairs of contiguous mica plates arranged successively at a distance from each other. Simulation of a microwave filter based on a Fabry-Perot resonator for microwave diagnostics of Doppler reflectometry, made it possible to construct an experimental filter sample with characteristics close to the given ones. The report presents the results of the development, modeling and experimental study of a filter based on a Fabry-Perot (FP) resonator. The introduction of the filter system made it possible to successfully carry out experimental measurements of the spectra of DR in the L-2M stellarator with an ECR heating power density of up to $3.4 \mathrm{MW} / \mathrm{m}^{3}$. The aim of the research was to create conditions for the stable operation of DR in conditions of powerful heating ECR radiation. To reduce the effect of an external signal with a frequency of $75.3 \mathrm{GHz}$ and noise in the range $60-80 \mathrm{GHz}$, it was necessary to create a new filtering system. In the filtration system we used band-stop filters. The task of these filters is to provide large attenuation (suppression) in a certain frequency range $\Delta \mathrm{f}$ near the central frequency $\mathrm{f}_{0}$.

The system of filters for diagnostics of DR consists of three pin-waveguide filters and filter based on FabryPerot resonator made of mica plates. In view of the design features of the diagnostics (in particular, the overall dimensions of the waveguide paths) the installation of additional pin filters, was not possible. It was required to design and manufacture a compact band-stop filter with a central suppression frequency of $75 \mathrm{GHz}$. A filter was proposed from the sequence of Fabry-Perot resonators. Millimeter and submillimeter filters based on the FabryPerot resonator are actively used in various diagnostics and radio astronomy. We used Fabry-Perot resonators with flat round mica plates (dielectric permittivity of mica $\varepsilon=6$ ) with diameter $\mathrm{D}=80 \mathrm{~mm}$ and thickness $\mathrm{S}=0.1 \mathrm{~mm}$.

The 3D-model (XYZ coordinate system) of the resonator filter was created in the computer-aided design system Electro-Magnetic Professional (CAD EMPro) from Keysight Technologies (formerly Agilent Technologies) on the fig. 1 .

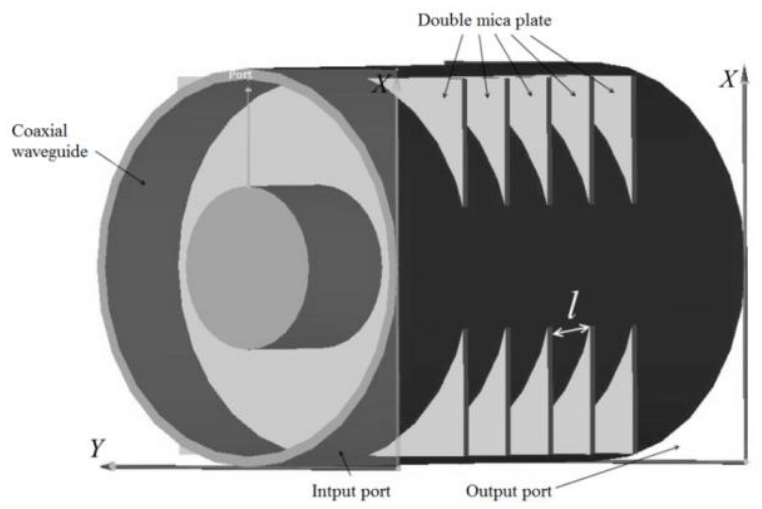

Fig. 1. The 3D-model of the filter based on a Fabry-Perot resonator

The band-stop filter with Fabri-Perot resonators is represented as a pair of mica plates in contact, located in succession at a distance $l$ from each other. The plates are placed in a coaxial waveguide perpendicular to its axis to ensure the propagation of the TEM wave. The calculation was carried out by the Finite Element Method (FEM) in the Agilent FEM Simulator block [4], the boundary conditions at the edges of the counting area are given by Perfect Matching Layer (PML). Waveguide ports are on both sides of the filter. Test measurements showed an improvement in signal/noise ratio.

The filter characteristics were measured using an MVNA-8-350 vector network analyzer from AB Millimetre with a measurement step of $10 \mathrm{kHz}$.

The level of loses (or transmission coefficient S21) of the waveguide-pin filters measured in the ranges 29-41 GHz (operation frequencies of DR) and 62.5-100 GHz. The transmission coefficients of the pin filter do not exceed $0.5 \mathrm{~dB}$ in the operating frequency range of the Doppler reflectometer and for the ECR frequency of the heating radiation $(75.3 \mathrm{GHz})$ more than $30 \mathrm{~dB}$. 
Taking into account the fact that three waveguide filters are used in the filtration system one common and one in each detector channel, the suppression of the heating frequency of $75.3 \mathrm{GHz}$ in the waveguide section of the filtration system should be about $60 \mathrm{~dB}$.

As can be seen on fig. 2 the level of noises in diagnostic signals were much reduced when FP filter was installed. This reduction of microwave heating in diagnostic channels allows to reliably measurement characteristics of plasmas fluctuations.

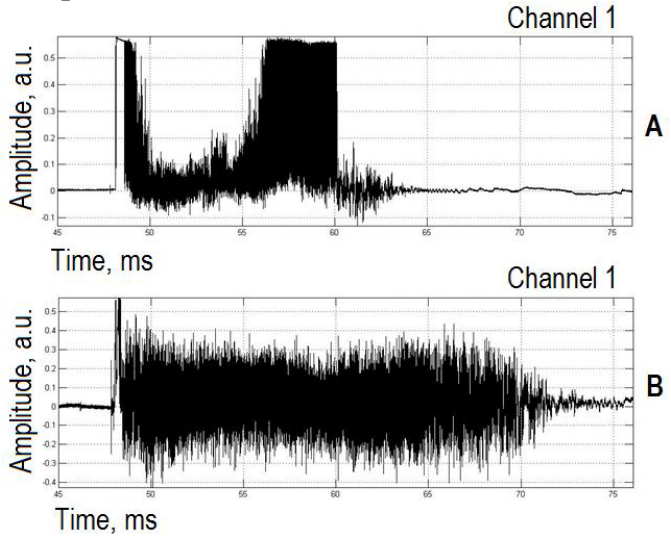

Fig. 2. Signals of Doppler reflectometry system (A) without FP filter; (B) - with installation of FP filter

As a result of the calculations, the transmission coefficient was obtained for the Fabry-Perot filter model. The results for the optimized model are shown in figure 3 in the form of a black solid curve in the range $40-80 \mathrm{GHz}$. As can be seen, at a frequency of $75.3 \mathrm{GHz}$, the attenuation value $\mathrm{S} 21=-21.9 \mathrm{~dB}$ was obtained.

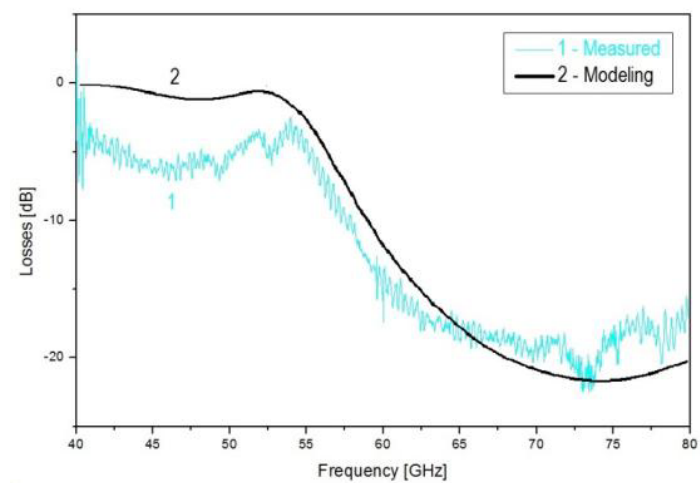

Fig. 3. The level of losses in the filter based on the Fabry-Perot resonator. Graph 1 - Measured value of filter attenuation, Graph 2 - Calculated value of attenuation of the filter model

It is worth noting that the calculated attenuation in the optimized filter model at 35 and $40 \mathrm{GHz}$ does not exceed $-0.9 \mathrm{~dB}$. Besides on the figure 3 shows the measured transmission coefficient of a filter based on a FabryPerot resonator. Due to the specifics of the analyzer device, data can be removed in discrete spectral ranges. It should be taken into account that all measurements were carried out with the continuous generation of low-power radiation in MVNA-8-350 vector network analyzer of the order of hundreds of $\mathrm{mW}$.

The developed and created filter, as well as the antenna system, can be used for microwave diagnostics (scattering, interferometry, reflectometry) on toroidal installations (tokamaks and stellarators) with electron cyclotron heating.

Optimization of algorithms for data analysis and the development of new software represented in [5].

The algorithm for analyzing the DR data includes the procedure for preparing data, analyzing data, visualizing and storing the results of the analysis. As part of optimizing the diagnosis of DR, the hardware and software parts were upgraded. As part of the hardware, a transition was made to the allotted ADC ( 2 channels, $100 \mathrm{MHz}, 12 \mathrm{bit}$ ). The data transfer from the ADC is done via Ethernet over fiber optic communication. Data is stored on a NAS (Network Attached Storage) with access rights. Calculations are performed by operators on personal computers (Matlab) and on a computer server (Python, CUDA). The preprocessing procedure includes: converting the output format, normalizing the signal, searching for features in the time series. The procedure for analyzing data in general form is described in [1]. An optimized algorithm for analyzing data and searching for the velocity of poloidal rotation of a plasma column includes methods for automatically calculating a set of parameters. Such parameters are: the evolution of statistical moments, the search for the maximum Doppler shift (different algorithms), spectral analysis (Fourier and wavelet) and other parameters of signals that carry a physical meaning. Using these parameters allows you to work with such a concept as feature. After feature extraction we can talk about the application of methods of machine learning for the analysis of plasma density fluctuations.

This work was supported in part by the support of the Russian President's grant for young scientists \#MK5298.2016 .8

\section{References}

1. Pshenichnikov, A.A., Kolik, L.V., Malyh, N.I., Petrov, A.E. et al., The use of Doppler reflectometry in the L-2M stellarator // Plasma Physics, 2005. Vol 31. № 7. Pp. 604-611.

2. Batanov, G.M., Harchev, N.K., Skvortsova, N.N., Sarksian, K.A. et al., New gyrotron complex MiG-3 for creating and plasma heating in the L-2M stellarator and the results of the first experiments // Applied Physics. 2012. № 6. Pp. 79-87.

3. Kharchevskii, A.A., Bogachev, N.N., Malakhov, D.V., Optimization of doppler reflectometry system for operation in high energy input in the 1-2m stellarator // Engineering Physics, №6, 2016, Pp.59-65

4. Keysight Technologies. About EMPro. URL: http:// www.keysight.com/en/pc-1297143/EMPro.

5. https://github.com/malakhovdmitry/12m 